\title{
Model-independent test of the FLRW metric, the flatness of the Universe, and non-local estimation of $H_{0} r_{\mathrm{d}}$
}

\author{
Benjamin L'Huillier $^{a}$ and Arman Shafieloo ${ }^{a, b}$ \\ ${ }^{a}$ Korea Astronomy and Space Science Institute, Yuseong-gu, 776 Daedeok daero, Daejeon, \\ Korea \\ ${ }^{b}$ University of Science and Technology, Yuseong-gu 217 Gajeong-ro, Daejeon, Korea \\ E-mail: benjamin@kasi.re.kr, shafieloo@kasi.re.kr
}

\begin{abstract}
Using measurements of $H(z)$ and $d_{\mathrm{A}}(z)$ from the Baryon Oscillation Spectroscopic Survey (BOSS) DR12 and luminosity distances from the Joint Lightcurve Analysis (JLA) compilation of supernovae (SN), we measure $H_{0} r_{\mathrm{d}}$ without any model assumption. Our measurement of $H_{0} r_{\mathrm{d}}=\left(10033.20_{-371.81}^{+333.10}(\mathrm{SN}) \pm 128.19(\mathrm{BAO})\right) \mathrm{km} \mathrm{s}^{-1}$ is consistent with Planck constrains for the flat $\Lambda \mathrm{CDM}$ model. We also report that higher expansion history rates $h(z)$ (among the possibilities) as well as lower-bound values of $H_{0} r_{\mathrm{d}}$ result in better internal consistency among the independent data $\left(H(z) r_{\mathrm{d}}\right.$ and $d_{\mathrm{A}}(z) / r_{\mathrm{d}}$ from BAO at $z=0.32$ and $z=0.57$ and $d_{\mathrm{L}}$ from JLA) we used in this work. This can be interpreted as an interesting and independent support of Planck cosmology without using any cosmic microwave background data. We then combine these observables to test the Friedmann-Lemaitre-Robertson-Walker (FLRW) metric and the flatness of the Universe in a model-independent way at two redshifts, namely 0.32 and 0.57 , by introducing a new diagnostic for flat-FLRW, $\Theta(z)$, which only depends on observables of $\mathrm{BAO}$ and SN data. Our results are consistent with a flat-FLRW Universe within $2 \sigma$.
\end{abstract}

Keywords: Cosmology, supernova type Ia - standard candles, baryon acoustic oscillations,

ArXiv ePrint: 1606.06832 


\section{Contents}

1 Introduction $\quad 1$

2 Method 2

2.1 Smoothing 2

2.2 Error propagation 3

3 Results 4

3.1 Smooth reconstruction 4

3.2 Estimation of $H_{0} r_{\mathrm{d}}$ and testing observational consistencies 5

$\begin{array}{lll}3.3 \text { Curvature test: } \Theta(z) \text { and } \mathcal{O}_{k}(z) & 7\end{array}$

\section{Introduction}

The current concordance model of the Universe relies on the important assumptions that the Universe is isotropic and homogeneous on large scale, and that gravity is described by General Relativity. Under these assumptions, the solution to Einstein's field equations is the Friedmann-Lemaitre-Robertson-Walker (FLRW) metric. Considering other aspects of the concordance model, such as assumption of the power-law form of the primordial spectrum and assuming cosmological constant as dark energy, we can make predictions on the behaviour of the Universe in different contexts and confront it with cosmological observations to constrain its six basic parameters. However, testing different assumptions of the standard model of cosmology, including its curvature and metric, is still of primordial importance [1-10].

Supernovae (SN) can be used to trace the expansion history of the Universe, and have revealed its recent acceleration [11-13]. In addition, the baryonic acoustic oscillations (BAO) can probe the growth of structures [14-16] by measuring $H(z) r_{\mathrm{d}}$ and $d_{\mathrm{A}}(z) / r_{\mathrm{d}}$, where

$$
r_{\mathrm{d}}=\frac{c}{\sqrt{3}} \int_{0}^{1 /\left(1+z_{\mathrm{drag}}\right)} \frac{\mathrm{d} a}{a^{2} H(a) \sqrt{1+\frac{3 \Omega_{\mathrm{b}}}{4 \Omega_{\mathrm{r}}}}},
$$

is the sound horizon at the drag epoch, and $\Omega_{\mathrm{b}}$ and $\Omega_{\mathrm{r}}$ are the baryon and radiation density parameters at $z=0$.

In this work we use a non-parametric approach to derive $H_{0} r_{\mathrm{d}}$, combining independent data from supernovae and baryon acoustic oscillations, and test the consistency of the results. Using some local measurements of $H_{0}$ we can then estimate the value of $r_{\mathrm{d}}$ in a non-parametric and model-independent manner. Measuring $r_{\mathrm{d}}$ model-independently avoids to bias the results toward a particular cosmological model $[17,18]$. We should also note that in our paper we use a non-parametric approach to reconstruct the expansion history of the universe and this makes our results even free from assuming any particular parametric form. We then test the FLRW metric and flatness of the Universe using a non-parametric and model-independent approach depending on the observables directly.

Section 2 describes the method used in this study, and the results are presented in $\S 3$. In $\S 4$ we discuss about our results and conclude. 


\section{Method}

In a FLRW metric, the curvature parameter $\Omega_{k}$ is constant with redshift (its associated energy varies like $\left.\Omega_{k}(1+z)^{2}\right)$ and the luminosity distance can be written as [19]:

$$
d_{\mathrm{L}}(z)=(1+z)^{2} d_{\mathrm{A}}(z)=\frac{(1+z) d_{\mathrm{H}}}{\sqrt{-\Omega_{k}}} \sin \left(\sqrt{-\Omega_{k}} \int_{0}^{z} \frac{\mathrm{d} z^{\prime}}{h\left(z^{\prime}\right)}\right),
$$

where

$$
d_{\mathrm{H}}=\frac{c}{H_{0}}
$$

is the Hubble distance, $d_{\mathrm{A}}$ is the angular diameter distance, and

$$
h^{2}(z)=\Omega_{\mathrm{m}}(1+z)^{3}+\Omega_{k}(1+z)^{2}+\Omega_{\mathrm{DE}} \exp \left(3 \int_{0}^{z} \frac{1+w\left(z^{\prime}\right)}{1+z^{\prime}} \mathrm{d} z^{\prime}\right)
$$

for a FLRW universe where the equation of state of dark energy is $w(z)=P / \rho(w(z)=-1$ for the cosmological constant). It is also useful to work with the dimensionless comoving distance defined as

$$
\mathcal{D}(z)=\frac{1}{(1+z) d_{\mathrm{H}}} d_{\mathrm{L}}(z)=\frac{1}{\sqrt{-\Omega_{k}}} \sin \left(\sqrt{-\Omega_{k}} \int_{0}^{z} \frac{\mathrm{d} z^{\prime}}{h\left(z^{\prime}\right)}\right) .
$$

The main idea here - to test the curvature, FLRW metric, estimate $H_{0} r_{\mathrm{d}}$, and test the internal consistency between different observations - is to obtain $\mathcal{D}(z)-\mathcal{D}^{\prime}(z)$ and $d_{\mathrm{A}}(z) / r_{\mathrm{d}}$ $H(z) r_{\mathrm{d}}$ from independent measurements without assuming a cosmological model. In order to get $\mathcal{D}^{\prime}(z)$, we can evaluate $\mathcal{D}(z)$ at all redshifts using the distance modulus of supernovae and calculate its derivative. In this work, we use the JLA sample [20]. In [3, 21, 22], the authors introduced a non-parametric method to reconstruct the luminosity distances from supernovae data by iteratively smoothing the residuals. The log-normal smoothing kernel and iterative approach used in $[3,21,22]$ has shown the effectiveness of the method in direct reconstruction as it has already been implemented succesfully in different contexts. Non-parametric methods can be very useful because they are model-independent, which enables them to look for unexpected features in the data beyond the flexibility of parametric approaches. The actual quantity recovered by this method is [3]

$$
d_{\mathrm{L}}^{\mathrm{rec}}(z)=H_{0} / c d_{\mathrm{L}}(z)=(1+z) \mathcal{D}(z) .
$$

On the other hand, $d_{\mathrm{A}}(z) / r_{\mathrm{d}}$ and $H(z) r_{\mathrm{d}}$ can be measured directly by the baryonic acoustic oscillations, for instance from the Baryon Oscillation Spectroscopic Survey (BOSS) DR12 [15]. We combine all these results for the purposes of this work.

\subsection{Smoothing}

In our procedure we go through the following steps:

1. Start with different initial guess models,

2. Iteratively smooth the data with a kernel similar to what has been proposed before, using $N_{\text {iter }}$ iterations, 
3. Only keep those reconstructions that yield a better $\chi^{2}$ than a reference model.

We use the best-fit flat $\Lambda \mathrm{CDM}$ as our reference model. Note that we do not consider these reconstructions to be necessarily more probable than the reference model. We use the reference model only as a criterion to accept a non-exhaustive sample of reconstructions with plausible and probable expansion histories.

In order to explore the allowed $\mathcal{D}$, we start with different initial guesses: best-fit flat$\Lambda \mathrm{CDM}$ open $\Lambda \mathrm{CDM}, w \mathrm{CDM}$, standard CDM $\left(\Omega_{\mathrm{m}}=1\right)$, open CDM $\left(\Omega_{\mathrm{m}}+\Omega_{k}=1\right)$, empty universe $\left(\Omega_{k}=1\right)$, de Sitter universe $\left(\Omega_{\Lambda}=1\right)$, as well as flat- $\Lambda$ CDM universes with $\Omega_{\mathrm{m}}=$ $0.1, \ldots, 0.9$.

We followed $[3,21,22]$ and used a log-normal kernel to smooth the supernovae data, taking into account the errors in $\mu$. We start from an initial guess $\hat{\mu}_{0}\left(z_{i}\right)$ at the input data $z_{i}$ (see previous section). The smooth distance modulus at iteration $n+1$ is then calculated by

$$
\begin{aligned}
\hat{\mu}_{n+1}(z) & =\hat{\mu}_{n}(z)+N(z) \sum_{i}\left(\frac{\mu\left(z_{i}\right)-\hat{\mu}_{n}\left(z_{i}\right)}{\sigma_{i}^{2}} \exp \left(-\frac{\ln ^{2}\left(\frac{1+z_{i}}{1+z}\right)}{2 \Delta^{2}}\right)\right) \\
N^{-1}(z) & =\sum_{i}\left(\frac{1}{\sigma_{i}^{2}} \exp \left(-\frac{\ln ^{2}\left(\frac{1+z_{i}}{1+z}\right)}{2 \Delta^{2}}\right)\right),
\end{aligned}
$$

where $\hat{\mu}_{n}(z)$ is the reconstructed distance modulus at any redshift $z$, while $\mu\left(z_{i}\right)$ and $\sigma_{i}$ are the measured distance modulus and its associated error at redshift $z_{i}$; and $N(z)$ is a normalisation factor. At each iteration, we calculate the $\chi^{2}$ defined as

$$
\chi_{n}^{2}=\left(\mu-\hat{\mu}_{n}\right)^{\mathrm{T}} \mathbf{C}^{-1}\left(\mu-\hat{\mu}_{n}\right)
$$

where $\mathbf{C}$ is the data covariance matrix.

Note that the supernovae data provide us with $\mu(z)=5 \log _{10} d_{\mathrm{L}}(z)+25=m(z)-\mathcal{M}$, which can be rewritten as $\mu(z)=5 \log _{10}\left(c d_{\mathrm{L}}(z) / H_{0}\right)+\mu_{0}$, where $d_{\mathrm{L}}(z)$ is expressed in Mpc, and $\mathcal{M}$ is the absolute magnitude.

Following [3], we used $\Delta=0.3$ since the number of supernovae and their uncertainties are of the same order as in [3]. In practice, we used $N_{\text {iter }}=200$, and keep only iterations that yield a better $\chi^{2}$ than the reference model. We used the best-fit flat $\Lambda \mathrm{CDM}$ as our reference model. This leaves us with about 1600 trajectories of $\mathcal{D}$ with better $\chi^{2}$ than the reference model.

In order to obtain $\mathcal{D}^{\prime}(z)=\mathrm{d} \mathcal{D}(z) / \mathrm{d} z=\mathrm{d}\left(d_{\mathrm{L}}^{\text {rec }}(z) /(1+z)\right) / \mathrm{d} z$, we numerically calculate the derivative of $\mathcal{D}$ and also impose $\mathcal{D}^{\prime}(z=0)=1$.

\subsection{Error propagation}

To combine the results from smoothing with the BAO data, we propagate the errors to combine the uncertainties from reconstruction of the expansion history from supernovae data and the uncertainties of the $H(z)$ from BAO data. The errors on BAO $\left(H(z) r_{\mathrm{d}}\right.$ and $\left.d_{\mathrm{A}} / r_{\mathrm{d}}\right)$ are propagated in the same way as in [3], taking into account the correlation between them, and adding external sources (such as $H_{0}$ ) when needed. The errors due to the reconstructions from the SNIa data are taken into account in the following way. We the median as the central value, and we used the minimum and maximum derived values at each redshift to set the errors. Therefore we quote two kinds of errors of different nature, namely SN and BAO. We 


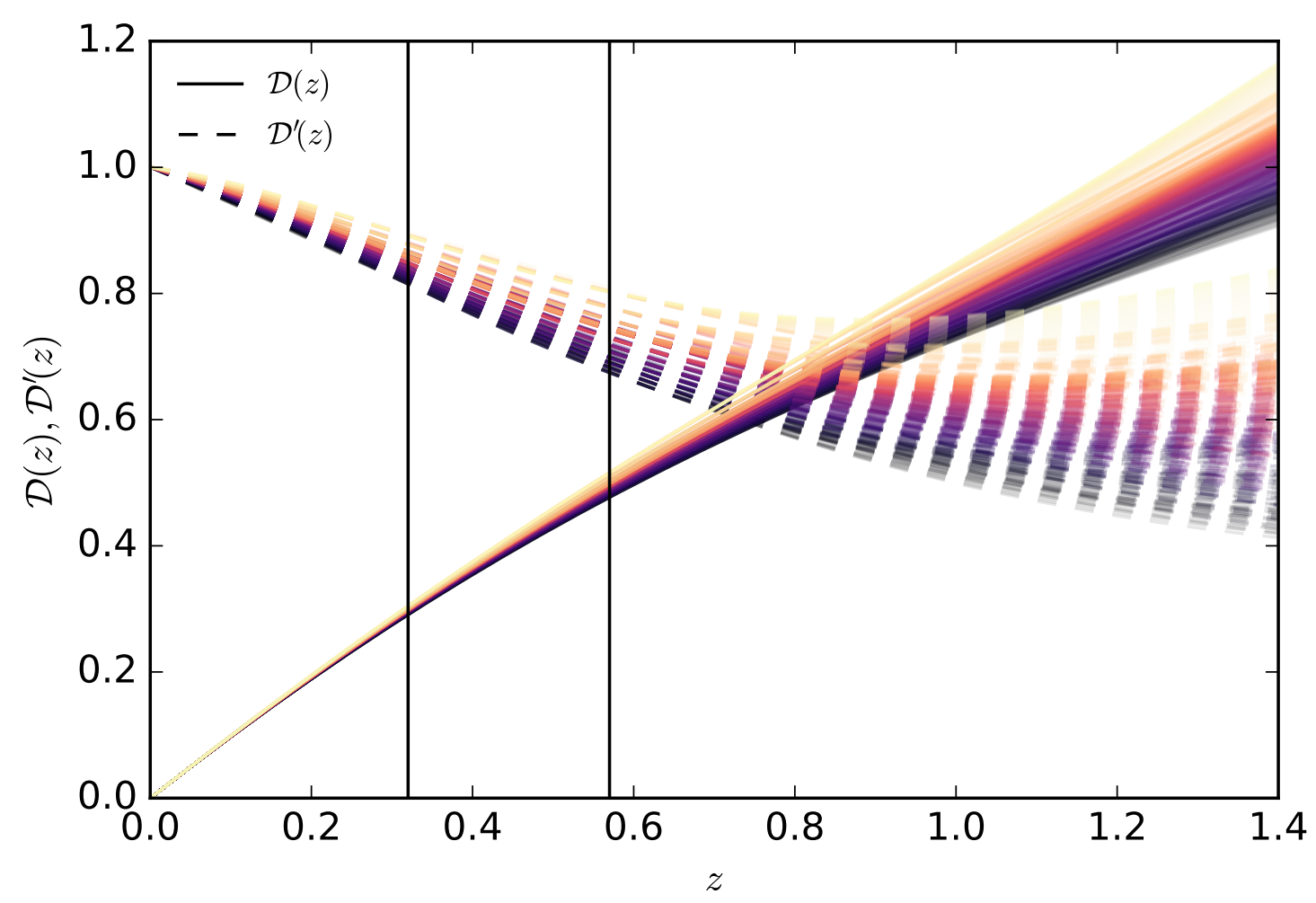

Figure 1: Reconstructed $\mathcal{D}(z)$ (solid lines) and $\mathcal{D}^{\prime}(z)$ (dashed lines) from JLA supernovae compilation. All lines shown here have a better $\chi^{2}$ than the reference model (best-fit flat $\Lambda \mathrm{CDM}$ ). Colors are used to show different reconstructed $\mathcal{D}(z)$ and their corresponding (correlated) $\mathcal{D}^{\prime}(z)$.

should note here again that all reconstructions used here have a better likelihood than the best-fit flat- $\Lambda \mathrm{CDM}$ model (our reference model). In other words, the results derived in this work represent possibilities that all have reasonable likelihood (and better than the best-fit $\Lambda \mathrm{CDM}$ model) given the data.

\section{Results}

\subsection{Smooth reconstruction}

Figure 1 shows the reconstructed $\mathcal{D}$ (solid lines) and $\mathcal{D}^{\prime}$ (dashed lines) for several initial guesses. All the curves shown here have a better $\chi^{2}$ than the reference model (best-fit $\Lambda \mathrm{CDM}$ ). For the sake of visualization, we only plotted every fourth reconstruction. We colour-coded the curves (according to their value of $\mathcal{D}(z)$ at $z=1.4$ ) since each reconstructed $\mathcal{D}(z)$ has its own corresponding (correlated) $\mathcal{D}^{\prime}(z)$. The agreement in $\mathcal{D}$ at low- $z$ is very good, owing to the large number of data points, while at higher redshifts the constraints are less strong and different models start to deviate from each other. In our range of interest, $z=0.32-0.57$, the agreement is of the order of $1 \%$. However, $\mathcal{D}$ ' shows some stronger deviation for different models which is expected considering it to be a derivative of $\mathcal{D}$. 


\subsection{Estimation of $H_{0} r_{\mathrm{d}}$ and testing observational consistencies}

In the previous section, we reconstructed $\mathcal{D}^{\prime}(z)$ at any redshift from the supernovae data. Under assumption of flatness (eq. 2.4), we have $h(z)=1 / \mathcal{D}^{\prime}(z)$. We can combine it with $H(z) r_{\mathrm{d}}$ obtained from the radial mode of the BAO to estimate

$$
H_{0} r_{\mathrm{d}}=\frac{H(z) r_{\mathrm{d}}}{h(z)}=H(z) r_{\mathrm{d}} \mathcal{D}^{\prime}(z) .
$$

We will refer to this as method A.

Alternatively, combining eqs. (2.1) and (2.4), we can write

$$
H_{0} r_{\mathrm{d}}=\frac{c \mathcal{D}(z) r_{\mathrm{d}}}{(1+z) d_{\mathrm{A}}(z)},
$$

where $d_{\mathrm{A}}(z) / r_{\mathrm{d}}$ comes from the transverse $\mathrm{BAO}$ [15]. We can calculate $H_{0} r_{\mathrm{d}}$ from the data at the two redshifts of LOWZ and CMASS. The main interest of this measurement is the very high accuracy of $d_{\mathrm{A}}(z)$. Moreover, this estimation of $H_{0} r_{\mathrm{d}}$ does not assume flatness, since $\mathcal{D}(z)$ is directly obtained from the smoothing. This method will be referred to as method B.

We can thus derive $H_{0} r_{\mathrm{d}}$ but also, we can test the internal consistency of our measurements where the derived $H_{0} r_{\mathrm{d}}$ from method $\mathrm{A}$ and $\mathrm{B}$ at both redshifts (0.32 and 0.57) should result in consistent values.

Figure 2 shows the value of $H_{0} r_{\mathrm{d}}$ from LOWZ ( $x$-axis) versus that from CMASS ( $y$-axis) for method A (squares) and B (circles). Each point is given by one reconstruction of $\mathcal{D}(z)$ with $\chi^{2}<\chi_{\text {ref, }}^{2}$, while the error-bars come from the BAO. The colour-code is the same as that of the expansion history reconstruction shown in Fig. 1. One should note that results shown with the same colours should be consistent with each other. The black, diagonal line shows the loci where $H_{0} r_{\mathrm{d}}$ from CMASS and LOWZ are equal, and the green square shows the $\pm 1 \sigma$ region centred around the Planck best-fit value.

Method A (squares) has larger error bars, due to the larger errors on $H(z)$ and also larger dispersion of $D^{\prime}(z)$. The reconstructions yielding a lower value of $H_{0} r_{\mathrm{d}}$ (black colour) are consistent between CMASS and LOWZ, while values yielding larger $H_{0} r_{\mathrm{d}}$ (yellow) show inconsistency. Method B has smaller error bars, and larger values of $H_{0} r_{\mathrm{d}} \simeq 10200 \mathrm{~km} \mathrm{~s}^{-1}$ are consistent between LOWZ and CMASS, while lower values are slightly in tension.

However, when considering both methods simultaneously, reconstructions yielding higher values of $H_{0} r_{\mathrm{d}}$ (yellow points) appear to be in tension between the two methods. On the contrary, lower values of $H_{0} r_{\mathrm{d}} \simeq 9800 \mathrm{~km} \mathrm{~s}^{-1}$ are preferred combining results of both methods at both redshifts.

We also calculated the best-fit value of $H_{0} r_{\mathrm{d}}$ using the flat- $\Lambda \mathrm{CDM}$ Markov chains (TT, TE, EE, LowP, and lensing) from [23]:

$$
H_{0} r_{\mathrm{d}}=(9944.0 \pm 127.4) \mathrm{km} \mathrm{s}^{-1},
$$

and we show it as a green square. The lower values of $H_{0} r_{\mathrm{d}}$ are consistent with these results, while the higher value, inconsistent together, are also inconsistent with Planck.

We then calculated the weighted average of $H_{0} r_{\mathrm{d}}$ over the two redshifts for each reconstruction for methods A and B, and reported the median value in table 1 . The third column is the error due to the smoothing of the SN data, given by the minimal and maximal $H_{0} r_{\mathrm{d}}$ over all reconstructions, and the fourth column shows the errors due to BAO. The results 


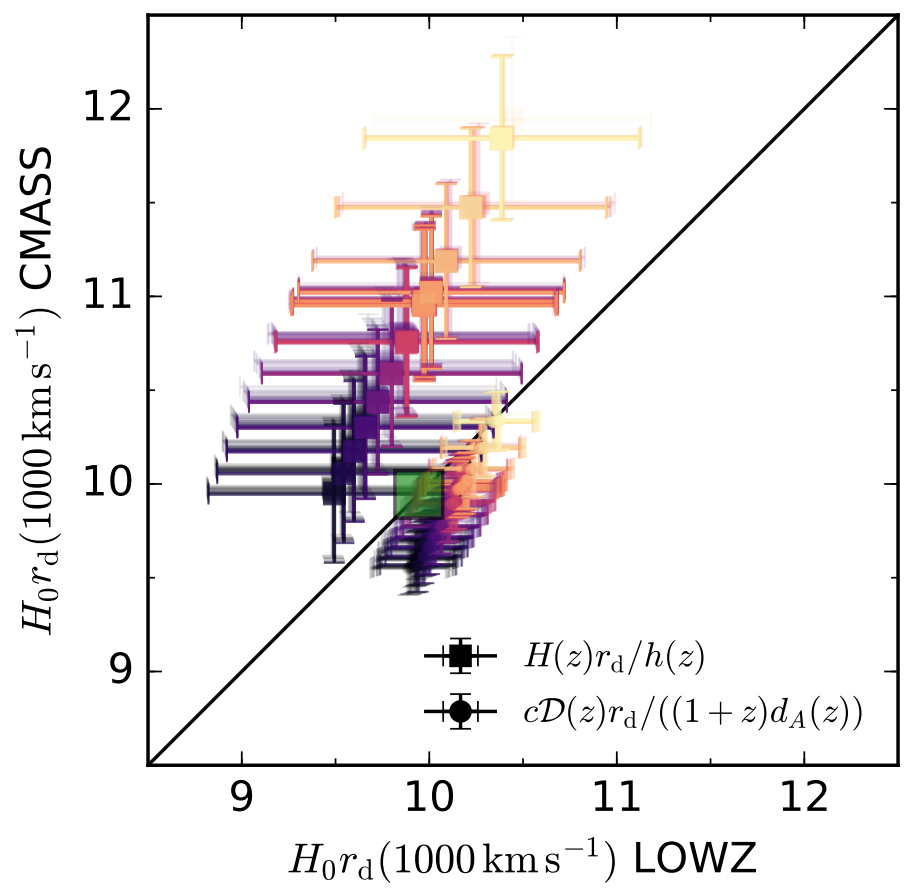

Figure 2: $H_{0} r_{\mathrm{d}}$ from LOWZ versus CMASS from methods A (squares) and B (dots). The green square at $\left(10^{4}, 10^{4}\right) \mathrm{km} \mathrm{s}^{-1}$ shows the $\pm 1 \sigma$ region centred around the Planck 2015 bestfit flat- $\Lambda$ CDM value for $H_{0} r_{\mathrm{d}}$. Colour code is the same as Fig. 1 for different reconstructions of the expansion history from supernovae data. One should note that there is a better consistency between all measurements for lower-bound values of $H_{0} r_{\mathrm{d}}$ and higher rate expansion histories (darker lines in Fig. 1).

Table 1: $H_{0} r_{\mathrm{d}}$ derived model-independently from Eq. (3.2) and (3.2). The second column is the median value of the weighted average of the measurements at $z=0.32$ and 0.57 , the third column is the error given by the supernovae, defined by the minimum and maximum values of $H_{0} r_{\mathrm{d}}$ among all reconstructions, and the fourth column shows the errors given by the BAO.

\begin{tabular}{llll}
\hline$H_{0} r_{\mathrm{d}}$ & $\begin{array}{l}\text { Median } \\
\left(\mathrm{km} \mathrm{s}^{-1}\right)\end{array}$ & $\begin{array}{l}\text { Error (SN) } \\
\left(\mathrm{km} \mathrm{s}^{-1}\right)\end{array}$ & $\begin{array}{l}\text { Error (BAO) } \\
\left(\mathrm{km} \mathrm{s}^{-1}\right)\end{array}$ \\
\hline Method A & 10712.37 & ${ }_{-871.45}^{+837.90}$ & \pm 378.38 \\
Method B & 10033.20 & ${ }_{-371.81}^{+333.10}$ & \pm 128.19 \\
\hline
\end{tabular}

from both methods are consistent with the flat- $\Lambda \mathrm{CDM}$ model (eq. 3.3). Method B has smaller error-bars and does not assume flatness, therefore we chose it as our main result.

Using an external measurement of $H_{0}$, we can then estimate $r_{\mathrm{d}}$. Table 2 shows our estimation of $r_{\mathrm{d}}$ from method $\mathrm{B}$, for the two values of $H_{0}$ from Riess [24] (R16) and Rigault (R15) [25]. The medians and errors are estimated in the same way as those of $H_{0} r_{\mathrm{d}}$. The values of $r_{\mathrm{d}}$ obtained from $H_{0}$ from $\mathrm{R} 15$ are fully consistent the $\Lambda \mathrm{CDM}$ best-fit value of 
Table 2: Estimation of $r_{\mathrm{d}}$ assuming $H_{0}$ from R16 and R15. The errors are defined in the same way as table 1 .

\begin{tabular}{llll}
\hline$r_{\mathrm{d}}$ & $\begin{array}{l}\text { Median } \\
(\mathrm{Mpc})\end{array}$ & $\begin{array}{l}\text { Error }(\mathrm{SN}) \\
(\mathrm{Mpc})\end{array}$ & $\begin{array}{l}\text { Error }\left(\mathrm{BAO}+H_{0}\right) \\
(\mathrm{Mpc})\end{array}$ \\
\hline R16, Method B & 137.40 & ${ }_{-5.09}^{+4.56}$ & \pm 3.88 \\
R15, Method B & 142.11 & ${ }_{-5.27}^{+4.72}$ & \pm 5.69 \\
\hline
\end{tabular}

$r_{\mathrm{d}}=(147.41 \pm 0.30) \mathrm{Mpc}$ [23], while with $H_{0}$ from R16, the results are consistent within $2 \sigma$. In spite of the large error-bars, our approach gives a fully model-independent estimation of $r_{\mathrm{d}}$ where we even used a non-parametric approach in reconstruction of the expansion history. We should emphasize here that the tight constraints on $H_{0} r_{\mathrm{d}}$ for the $\Lambda \mathrm{CDM}$ model are due to parametric nature of data fitting. Otherwise, our reconstructions all have better likelihood to the data than the best-fit $\Lambda \mathrm{CDM}$ model.

\subsection{Curvature test: $\Theta(z)$ and $\mathcal{O}_{k}(z)$}

The $\mathcal{O}_{k}(z)$ diagnostic $[1,3,7,10]$ was introduced to test the FLRW metric as well as the curvature of the Universe.

$$
\mathcal{O}_{k}(z)=\frac{\left(h(z) \mathcal{D}^{\prime}(z)\right)^{2}-1}{\mathcal{D}^{2}(z)}
$$

In a FLRW metric, $\mathcal{O}_{k}(z)$ is constant and equal to $\Omega_{k}$. In a flat-FLRW metric, one has $\mathcal{O}_{k}(z)=0$, or

$$
\Theta(z) \equiv h(z) \mathcal{D}^{\prime}(z)=\frac{H(z)}{H_{0}} \mathcal{D}^{\prime}(z)=1 .
$$

Therefore, $\mathcal{O}_{k}$ can be seen as a curvature test, and (if consistent with a constant value), gives the curvature density parameter, while $\Theta(z)=1$ is a yes/no test to flat-FLRW.

By noticing that $h(z)=H(z) r_{\mathrm{d}} / H_{0} r_{\mathrm{d}}$, one can combine the BAO data with $H_{0} r_{\mathrm{d}}$ calculated from eq. (3.2) in $\S 3.2$. Both diagnostics can then be rewritten fully in terms of observables, as

$$
\begin{aligned}
\Theta(z) & =\frac{1+z}{c}\left(H(z) r_{\mathrm{d}} \frac{d_{\mathrm{A}}(z)}{r_{\mathrm{d}}}\right)\left(\frac{\mathcal{D}^{\prime}(z)}{\mathcal{D}(z)}\right), \\
& =F_{\mathrm{AP}}(z)\left(\frac{\mathcal{D}^{\prime}(z)}{\mathcal{D}(z)}\right) \\
\mathcal{O}_{k}(z) & =\frac{\Theta^{2}(z)-1}{\mathcal{D}^{2}(z)},
\end{aligned}
$$

where $\mathcal{D}$ and $\mathcal{D}^{\prime}$ are obtained by smoothing method (§2.1), $H(z) r_{\mathrm{d}}$ and $d_{\mathrm{A}}(z) / r_{\mathrm{d}}$ are given by the radial and tranverse mode of the $\mathrm{BAO}[15]$, and $F_{\mathrm{AP}}(z)=(1+z) d_{\mathrm{A}}(z) / c(z)$ is the Alcock-Pazcynski anisotropy parameter. We stress that in eq. (3.6), the first parenthesis depends on the BAO measurements, while the second one depends on the smooth supernovae data. In this new formulation, both statistics thus come entirely from the BAO and SN data, unlike in [3] where the authors also used $H_{0}$, and are fully model-independent. 

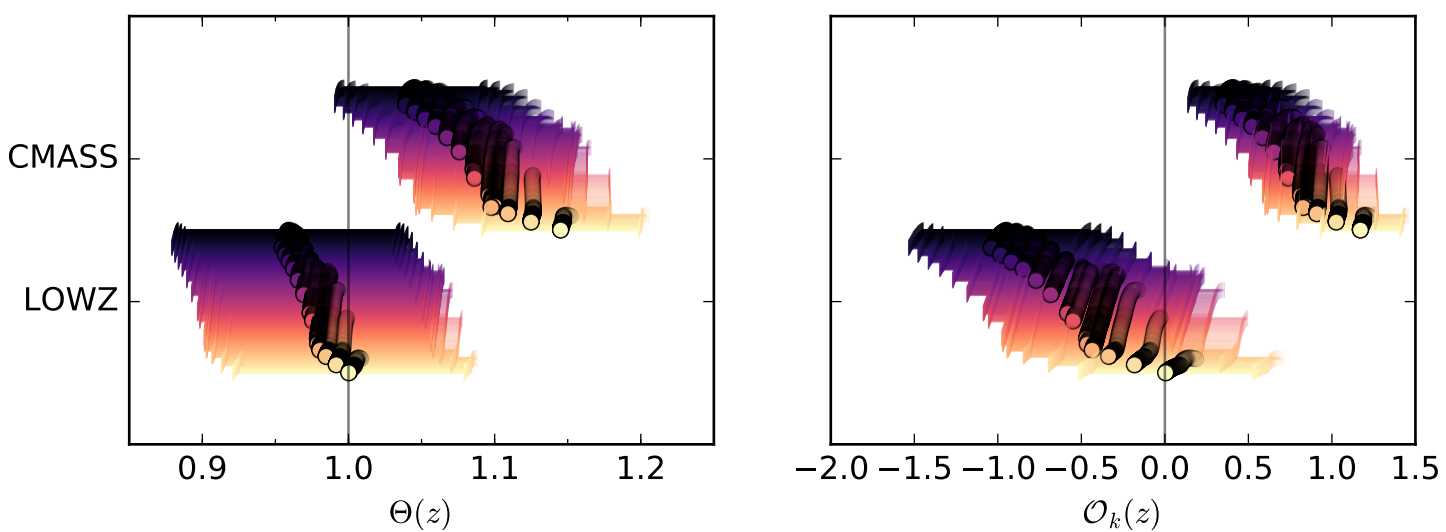

Figure 3: Curvature test. Left: $\Theta(z)=(1+z) / c\left(H(z) d_{\mathrm{A}}(z)\right)\left(\mathcal{D}^{\prime}(z) / \mathcal{D}(z)\right)$; right: $\mathcal{O}_{k}(z)$ (eqs. 3.4). Both statistics use $H(z) r_{\mathrm{d}}$ and $d_{\mathrm{A}}(z) / r_{\mathrm{d}}$ from BAO measurements, and $\mathcal{D}(z)$ and $\mathcal{D}^{\prime}(z)$ are obtained from the JLA supernovae data.

The left-hand panel of Fig. 3 thus shows $\Theta$ at the LOWZ and CMASS redshifts $(0.32$ and 0.57 ). Similarly to Fig. 2, each point corresponds to one reconstruction of the expansion history with $\chi^{2}<\chi_{\text {ref }}^{2}$, while the error-bars are coming from $H(z) r_{\mathrm{d}}$ and and $d_{\mathrm{A}}(z) / r_{\mathrm{d}}$, taking into account the correlation between $d_{\mathrm{A}}$ and $H$. The right-hand panel of Fig 3 shows $\mathcal{O}_{k}(z)$ at the LOWZ and CMASS redshifts. Here again, points in the same colour should be compared together. At $z=0.32, \Theta$ is consistent with one for each reconstruction. However, at $z=$ 0.57 , reconstructions with a lower rate of expansion history (yellow) show some tension with $\Theta=1$, while reconstructions with higher rate of expansion history (dark) are still consistent. Regarding $\mathcal{O}_{k}$, the reconstructions with higher expansion history show better consistency with a flat-FLRW universe at both redshifts. We should note that any inconsistency can be interpreted in two ways: (1) the metric is indeed not FLRW, and therefore $\mathcal{O}_{k}$ is not equal to $\Omega_{k}$. (2) There is some systematics in the supernovae and/or in the BAO data. If the metric is flat-FLRW, then $\Theta=1$ translate into

$$
H(z) d_{\mathrm{A}}(z)=\frac{1+z}{c} \frac{\mathcal{D}(z)}{\mathcal{D}^{\prime}(z)} .
$$

Therefore, an inconsistency between the two datasets can break the equality. One should note that it is possible to test this equality relation using only the observables of BAO and supernovae data taking into account all correlations.

It is interesting to notice we have reached an era where such litmus tests can be directly applied to the data to test the pillars of the concordance model with a reasonable precision. Future surveys will be able to bring down those errors, thus to improve the constrain on the flatness without parametric model assumptions.

\section{Discussion and conclusion}

Using the most recent BAO and supernovae data (BOSS DR 12 and JLA), we estimated $H_{0} r_{\mathrm{d}}$, where $r_{\mathrm{d}}$ is the sound horizon at the drag epoch, in a model-independent way. We calculated $H_{0} r_{\mathrm{d}}=\left(10033.20_{-371.81}^{+333.10}(\mathrm{SN}) \pm 128.19(\mathrm{BAO})\right) \mathrm{km} \mathrm{s}^{-1}$, which is consistent with the value from Planck $H_{0} r_{\mathrm{d}}=(9944.0 \pm 127.4) \mathrm{km} \mathrm{s}^{-1}$ for the concordance model. Without 
any assumption from the $\mathrm{CMB}$, and only using $\mathrm{BAO}$ and supernovae data, out results agree with the (model-dependent) best-fit value $H_{0} r_{\mathrm{d}}$ from Planck.

Using two astrophysical values of $H_{0}$, we derive $r_{\mathrm{d}}$ without using any parameterization and in a model-independent way. While the error-bars are large, the results using the Rigault measurement of $H_{0}$ are consistent with the Planck estimated value of the concordance model, while those using the Riess measurements show some tension with Planck.

We then tested the FLRW metric and the flatness of the Universe at redshifts 0.32 and 0.57 . We used $\mathcal{O}_{k}$ and introduced a new diagnostic $\Theta(z)=h(z) \mathcal{D}^{\prime}(z)$ to quantify departure from flatness and FLRW. For a flat-FLRW Universe, $\Theta(z)=1$. Our statistics can be fully written in terms of observables $\left(H(z) r_{\mathrm{d}}, d_{\mathrm{A}}(z) / r_{\mathrm{d}}, \mathcal{D}(z)\right.$ and $\left.\mathcal{D}^{\prime}(z)\right)$, and our test is therefore fully model-independent. We found some hints that there might be some inconsistency with flat-FLRW (Fig. 3), which may point to some systematics in the BAO or/and SN data or toward an actual departure from flatness. However, the results are still consistent with data fluctuations, therefore, better data are needed to conclude. Interestingly, the quality of the data has reached a level where we can consider such direct litmus tests very much plausible.

We should note here that there have been many articles in the literature using $H(z)$ derived from age of passively evolving galaxies through the cosmic chronometers approach [26-30]. However, to derive $H(z)$ through this approach we have to make strong assumptions on galaxy evolution characteristics. Therefore in our work we did not use any $H(z)$ data derived from age of passively evolving galaxies and our analysis is solely based on the BAO data from the BOSS survey.

Our internal consistency tests show some interesting results. While we expect to see a proper consistency between derived values of $H_{0} r_{\mathrm{d}}$ using LOWZ and CMASS data considering both $d_{A}(z)$ and $H(z)$ observations, we realized some considerable tensions. Having the expansion history directly derived from supernovae data we noticed that only by considering lower values of $H_{0} r_{\mathrm{d}}$ (around $10^{4} \mathrm{~km} \mathrm{~s}^{-1}$ and also considering larger expansion history rate (darker lines in Fig. 1 for $D(z)$ ), we can have consistent results between all measurements. This is particularly important looking at the results from CMASS data. This somehow supports the cosmological parameters from Planck concordance model cosmology (lower $H_{0}$ and higher $\Omega_{\mathrm{m}}$ ) without using the cosmic microwave background data. Future surveys, such as DESI, will measure $H(z) r_{\mathrm{d}}$ at several redshifts with smaller uncertainties. Using these values, we can test the flatness and the metrics in a wider range and with much higher precision and accuracy.

\section{Acknowledgement}

The authors thank Eric Linder for various useful discussions, and Changbom Park for his comments. A.S. would like to acknowledge the support of the National Research Foundation of Korea (NRF-2016922914). This work was supported by the National Institute of Supercomputing and Network/Korea Institute of Science and Technology Information with supercomputing resources including technical support.

\section{References}

[1] C. Clarkson, B. Bassett and T. H.-C. Lu, A General Test of the Copernican Principle, Physical Review Letters 101 (July, 2008) 011301, [0712.3457].

[2] D. L. Wiltshire, Average observational quantities in the timescape cosmology, Phys. Rev. D 80 (Dec., 2009) 123512, [0909.0749]. 
[3] A. Shafieloo and C. Clarkson, Model independent tests of the standard cosmological model, Phys. Rev. D 81 (Apr., 2010) 083537, [0911.4858].

[4] E. Mortsell and J. Jonsson, A model independent measure of the large scale curvature of the Universe, ArXiv e-prints (Feb., 2011) , [1102.4485].

[5] H. Wang and T.-J. Zhang, Constraints on Lemaître-Tolman-Bondi Models from Observational Hubble Parameter Data, ApJ 748 (Apr., 2012) 111, [1111.2400].

[6] Y.-L. Li, S.-Y. Li, T.-J. Zhang and T.-P. Li, Model-Independent Determination of Curvature Parameter Using $H(z)$ and $D_{A}(z)$ Data Pairs from BAO Measurements, ApJ 789 (July, 2014) L15, [1404.0773].

[7] D. Sapone, E. Majerotto and S. Nesseris, Curvature versus distances: Testing the FLRW cosmology, Phys. Rev. D 90 (July, 2014) 023012, [1402.2236].

[8] S. Räsänen, K. Bolejko and A. Finoguenov, New Test of the Friedmann-Lemaître-Robertson-Walker Metric Using the Distance Sum Rule, Physical Review Letters 115 (Sept., 2015) 101301, [1412.4976].

[9] Z.-S. Zhang, T.-J. Zhang, H. Wang and C. Ma, Testing the Copernican principle with the Hubble parameter, Phys. Rev. D 91 (Mar., 2015) 063506, [1210.1775].

[10] T. Buchert, A. A. Coley, H. Kleinert, B. F. Roukema and D. L. Wiltshire, Observational challenges for the standard FLRW model, International Journal of Modern Physics D 25 (Feb., 2016) 1630007-244, [1512.03313].

[11] S. Perlmutter, G. Aldering, M. della Valle, S. Deustua, R. S. Ellis, S. Fabbro et al., Discovery of a supernova explosion at half the age of the universe, Nature 391 (Jan., 1998) 51-+, [arXiv:astro-ph/9712212].

[12] A. G. Riess, A. V. Filippenko, P. Challis, A. Clocchiatti, A. Diercks, P. M. Garnavich et al., Observational Evidence from Supernovae for an Accelerating Universe and a Cosmological Constant, AJ 116 (Sept., 1998) 1009-1038, [arXiv:astro-ph/9805201].

[13] A. G. Riess, L.-G. Strolger, S. Casertano, H. C. Ferguson, B. Mobasher, B. Gold et al., New Hubble Space Telescope Discoveries of Type Ia Supernovae at $z>1$ : Narrowing Constraints on the Early Behavior of Dark Energy, ApJ 659 (Apr., 2007) 98-121, [arXiv:astro-ph/0611572].

[14] D. J. Eisenstein, I. Zehavi, D. W. Hogg, R. Scoccimarro, M. R. Blanton, R. C. Nichol et al., Detection of the Baryon Acoustic Peak in the Large-Scale Correlation Function of SDSS Luminous Red Galaxies, ApJ 633 (Nov., 2005) 560-574, [astro-ph/0501171].

[15] A. J. Cuesta, M. Vargas-Magaña, F. Beutler, A. S. Bolton, J. R. Brownstein, D. J. Eisenstein et al., The clustering of galaxies in the SDSS-III Baryon Oscillation Spectroscopic Survey: baryon acoustic oscillations in the correlation function of LOWZ and CMASS galaxies in Data Release 12, MNRAS 457 (Apr., 2016) 1770-1785, [1509.06371].

[16] H. Gil-Marín, W. J. Percival, A. J. Cuesta, J. R. Brownstein, C.-H. Chuang, S. Ho et al., The clustering of galaxies in the SDSS-III Baryon Oscillation Spectroscopic Survey: BAO measurement from the LOS-dependent power spectrum of DR12 BOSS galaxies, MNRAS (May, 2016) , [1509.06373].

[17] A. Heavens, R. Jimenez and L. Verde, Standard Rulers, Candles, and Clocks from the Low-Redshift Universe, Physical Review Letters 113 (Dec., 2014) 241302, [1409.6217].

[18] W. Sutherland, On measuring the absolute scale of baryon acoustic oscillations, MNRAS 426 (Oct., 2012) 1280-1290, [1205.0715].

[19] S. Weinberg, Gravitation and Cosmology: Principles and Applications of the General Theory of Relativity. July, 1972. 
[20] M. Betoule, R. Kessler, J. Guy, J. Mosher, D. Hardin, R. Biswas et al., Improved cosmological constraints from a joint analysis of the SDSS-II and SNLS supernova samples, A\&A 568 (Aug., 2014) A22, [1401.4064].

[21] A. Shafieloo, U. Alam, V. Sahni and A. A. Starobinsky, Smoothing supernova data to reconstruct the expansion history of the Universe and its age, MNRAS 366 (Mar., 2006) 1081-1095, [astro-ph/0505329].

[22] A. Shafieloo, Model-independent reconstruction of the expansion history of the Universe and the properties of dark energy, MNRAS 380 (Oct., 2007) 1573-1580, [astro-ph/0703034].

[23] Planck Collaboration, P. A. R. Ade, N. Aghanim, M. Arnaud, M. Ashdown, J. Aumont et al., Planck 2015 results. XIII. Cosmological parameters, A\&A 594 (Sept., 2016) A13, [1502.01589].

[24] A. G. Riess, L. M. Macri, S. L. Hoffmann, D. Scolnic, S. Casertano, A. V. Filippenko et al., A 2.4\% Determination of the Local Value of the Hubble Constant, ArXiv e-prints (Apr., 2016), [1604.01424].

[25] M. Rigault, G. Aldering, M. Kowalski, Y. Copin, P. Antilogus, C. Aragon et al., Confirmation of a Star Formation Bias in Type Ia Supernova Distances and its Effect on the Measurement of the Hubble Constant, ApJ 802 (Mar., 2015) 20, [1412.6501].

[26] R. Jimenez, L. Verde, T. Treu and D. Stern, Constraints on the Equation of State of Dark Energy and the Hubble Constant from Stellar Ages and the Cosmic Microwave Background, ApJ 593 (Aug., 2003) 622-629, [astro-ph/0302560].

[27] J. Simon, L. Verde and R. Jimenez, Constraints on the redshift dependence of the dark energy potential, Phys. Rev. D 71 (June, 2005) 123001, [astro-ph/0412269].

[28] E. Gaztañaga, A. Cabré and L. Hui, Clustering of luminous red galaxies - IV. Baryon acoustic peak in the line-of-sight direction and a direct measurement of H(z), MNRAS 399 (Nov., 2009) 1663-1680, [0807.3551].

[29] D. Stern, R. Jimenez, L. Verde, M. Kamionkowski and S. A. Stanford, Cosmic chronometers: constraining the equation of state of dark energy. I: H(z) measurements, J. Cosmology Astropart. Phys. 2 (Feb., 2010) 008, [0907.3149].

[30] M. Moresco, L. Pozzetti, A. Cimatti, R. Jimenez, C. Maraston, L. Verde et al., A 6\% measurement of the Hubble parameter at $z^{\sim} 0.45$ : direct evidence of the epoch of cosmic re-acceleration, J. Cosmology Astropart. Phys. 5 (May, 2016) 014, [1601.01701]. 